Théologiques

Théologiques

\title{
Caïn, protégé du Seigneur ?
}

\section{Les voix de Gn 4,1-16 dans une perspective narratologique}

\section{Alain Gignac}

Volume 17, numéro 2, 2009

Querelles d'images?

URI : https://id.erudit.org/iderudit/044065ar

DOI : https://doi.org/10.7202/044065ar

Aller au sommaire du numéro

\section{Éditeur(s)}

Faculté de théologie et de sciences des religions, Université de Montréal

ISSN

1188-7109 (imprimé)

1492-1413 (numérique)

Découvrir la revue

Citer cet article

Gignac, A. (2009). Caïn, protégé du Seigneur ? Les voix de Gn 4,1-16 dans une perspective narratologique. Théologiques, 17(2), 111-134.

https://doi.org/10.7202/044065ar
Résumé de l'article

La Bible interdit de représenter Dieu « en image » mais ne se prive pas de le mettre en scène dans des récits. Comment alors éviter que le lecteur ne se fabrique une " image de Dieu narrative " idolâtre ? À partir du récit de Caïn et Abel (Gn 4), analysé sous l'angle des dialogues et des points de vue différenciés des personnages, l'article démontre que la narration biblique, par la manière dont elle raconte, brouille la représentation de Dieu et invite à une lecture dialogique. Loin de la lecture moralisante et culpabilisante traditionnelle (qui reprend uniquement le point de vue de Caïn), on se rend compte que le récit insiste sur les multiples initiatives divines pour entrer en discussion avec Caïn afin de le faire advenir réellement à lui-même, comme individu moralement responsable. En introduction, une réflexion est proposée quant à l'intérêt de la narratologie pour évaluer le jeu des multiples relectures artistiques d'un récit biblique et pour établir un dialogue interdisciplinaire entre l'exégèse et l'histoire de l'art.
Ce document est protégé par la loi sur le droit d'auteur. L'utilisation des services d’Érudit (y compris la reproduction) est assujettie à sa politique d'utilisation que vous pouvez consulter en ligne.

https://apropos.erudit.org/fr/usagers/politique-dutilisation/ 
Théologiques 17, no 2 (2009) p. 111-134

\title{
Caïn, protégé du Seigneur?
}

\section{Les voix de Gn 4,1-16 dans une perspective narratologique}

\author{
Alain GIGNAC" \\ Théologie et sciences des religions \\ Université de Montréal
}

«Tu ne te feras aucune image sculptée, rien qui ressemble à ce qui est dans les cieux, là-haut, ou sur la terre, ici bas, ou dans les eaux, au-dessous de la terre " (Ex 20,4 traduction BJ 1998). Le Décalogue interdit la représentation du divin. La formulation elle-même a une facture totalisante (voire totalitaire), puisqu'elle couvre les «trois dimensions» de la cosmologie de Gn 1: le ciel, la terre et la mer, et semble étendre l'interdit à toute représentation, afin d'éviter toute dérive idolâtrique. En simplifiant (sans tenir compte des exceptions), on peut affirmer que le judaïsme et l'islam ont généralement suivi à la lettre l'interdit de la représentation, tandis que le christianisme l'a nuancé à partir d'un autre principe, celui de l'Incarnation.

En rappelant cela, mon propos n'est pas de revenir sur le problème de la portée théologique de l'image et de l'ambiguïté dont celle-ci est porteuse — d'autres articles de ce numéro abordent le problème. Il s'agit plutôt d'introduire ce questionnement: pourquoi la Bible, qui interdit l'image, se permet-elle de mettre Dieu en scène, de lui prêter des paroles, des sentiments, des intentions? Le récit biblique ne dépeint-il pas Dieu ? Pourquoi peut-on écrire sur Dieu (le représenter textuellement) mais ne peut-on pas le sculpter (le représenter) ? La narration biblique - avec un narrateur dont on

\footnotetext{
Alain Gignac est professeur agrégé à la Faculté de théologie et de sciences des religions de l'Université de Montréal. Ses recherches portent sur la narratologie appliquée aux textes discursifs du Nouveau Testament (Lettres aux Galates et aux Romains), ainsi que sur les lectures que font des lettres de Paul les philosophes politiques contemporains (Badiou, Agamben, etc.). Il a récemment publié (2009) "La mise en scène de $\mathrm{Rm}$ 7,7-8,4. Une approche narrative et synchronique ", dans U. Schnelle, dir., The Letter to the Romans. Colloquium Biblicum Lovaniense (Leuven, 25-27 juillet 2007), Leuven, Peeters (BETL; 226), p. 113-134.
}

(C) Revue Théologiques 2009. Tout droit réservé. 
oublie trop souvent l'outrecuidance inouie, lorsqu'il prétend par exemple être présent au commencement du monde - ne conduit-elle pas le lecteur à se construire une image de Dieu? Autrement dit, la Bible transgresse-t-elle son propre interdit?

Une réponse partielle à cette question énorme pourrait s'énoncer ainsi. La narration biblique, par la manière dont elle raconte, brouille la représentation de Dieu, ouvrant ainsi à une diversité de points de vue qui empêche le lecteur de se fabriquer un dieu à sa convenance. Avant de dire quelque chose sur Dieu, un texte biblique énonce comment ce dire est difficile, voire impossible. Plus précisément, avant d'apporter des réponses, il nous invite à vivre avec des questions. Or, pour reprendre les distinctions de Mikhail Bakhtine (1978; Jenny 2003), on a souvent tendance à réduire le récit à une lecture unique, monologique, sécurisante, certaine, plutôt que de mettre en valeur le caractère dialogique et polyphonique du texte.

Le texte massorétique du mythe de Caïn et Abel nous servira de test. Ce récit demeure énigmatique à bien des égards, défiant les certitudes interprétatives. Pourtant, la culture populaire et l'exégèse scientifique elle-même ont souvent proposé des lectures réductrices du récit. Par exemple, en hypertrophiant certains détails qui permettent de générer un discours moralisateur. Ou encore, en faisant du texte le simple miroir des rivalités entre sédentaires et nomades, dans une ligne étiologique. Or, si le texte n'est pas le lieu d'un seul discours qui deviendrait l'enjeu d'un conflit d'interprétation, mais bien le lieu même de ce conflit, nous ne pouvons imposer une seule interprétation au texte (lecture dogmatique), ni laisser le texte s'imposer à nous (lecture fondamentaliste). La métaphore du dialogue sousentend la possibilité d'un espace de liberté herméneutique. Comment les non-dits, les trous, les affirmations sibyllines du texte permettent-ils d'entendre d'autres voix, finalement occultées au fil du temps, au profit d'une voix qui aura su s'imposer? Comment rendre justice au caractère plurivoque (et donc équivoque) du récit? Comment repérer des chemins inédits parmi les possibles du texte, de manière à éviter les ornières, mais aussi à comprendre comment celles-ci se sont formées?

La question pourrait se reformuler ainsi: comment entendre toutes les voix du récit? J'entends avant tout suivre le parcours des personnages, au long des moments d'une intrigue divisée selon le modèle quinaire classique. Une attention spéciale sera portée aux voix du texte ainsi qu'aux dialogues - et donc aux points de vue successifs des personnages (incluant le narrateur, effacé mais dont les interventions s'avèrent décisives). Mais auparavant, je situerai mon étude dans une perspective d'interdisciplinarité et de 
Wirkungsgeschichte 1 - tant au plan théorique que pour une application au récit de Caïn et Abel.

\section{Problématique}

\subsection{En théorie: narratologie, effet de l'histoire et interdisciplinarité}

Les textes bibliques, et cela est particulièrement intense pour les récits mythiques de Genèse, subissent le travail de l'histoire (Wirkungsgeschichte). Pour chacun, on peut suivre la longue chaîne des interprétations, non seulement chez les commentateurs "autorisés ", mais surtout chez ceux que Bernard Sarrazin (1993) désigne comme les «braconniers», les artistes et penseurs qui viennent chasser sur le territoire théoriquement réservé aux théologiens. Le texte biblique est ainsi relu par les peintres, sculpteurs, romanciers, poètes, dramaturges, musiciens, philosophes ou essayistes... - et ceuxci se lisent ou se relisent entre eux, s'inspirant d'une œuvre qui les a précédés ou réagissant à celle-ci. Une généalogie des interprétations du texte se déploie alors, qui traverse la culture occidentale. Avec pour résultat que la «représentation » qu'on se fait d'un récit se trouve le plus souvent médiatisée par ses relectures, plutôt que de surgir d'un contact direct avec le texte. Éventuellement, il convient de s'interroger sur l'œuvre de ces braconniers: ont-ils proposé une lecture monologique ou dialogique du texte biblique?

Les catégories de Gérard Genette sur l'intertextualité permettent de clarifier ce phénomène de reprises incessantes ${ }^{2}$. Que ce soit sous un mode

1. Nous devons le terme Wirkungsgeschichte (littéralement, "histoire des effets") à Hans-Georg Gadamer et il est passé en exégèse comme synonyme d'histoire de l'interprétation, ce qui est quelque peu réducteur, voire constitue un contresens par rapport à la réflexion gadamérienne. La traduction proposée par Jean Grondin, «le travail de l'histoire", permet de restituer l'intuition herméneutique du concept: la réception d'un texte agit sur la compréhension que nous en avons et induit un travail d'enfantement, de mise au monde, du sens. Ce n'est pas tant le texte qui produit des effets dans l'histoire, que l'histoire qui permet d'actualiser des potentialités du texte, jusque là ignorées, car latentes.

2. À titre de rappel: Genette (1982, 5.45) trace la typologie suivante (tableau à gauche), qu'il raffine ensuite en proposant un continuum de six modes qu'il représente sous forme de rosette (tableau à droite):

\begin{tabular}{|l|l|l|l|}
\hline Régime: & $\bullet$ Ludique & $\bullet$ Satirique & $\bullet$ Sérieux \\
\hline Relation: & & & \\
\hline$\bullet$ Transformation & Parodie & Travestissement & Transposition \\
\hline$\bullet$ Imitation & Pastiche & Charge & Forgerie \\
\hline
\end{tabular}

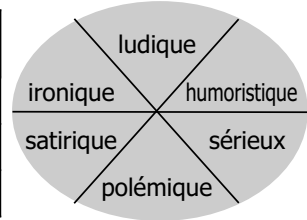


ludique, satirique ou sérieux, le texte biblique se trouve imité ou transformé, par phénomène d'ajouts, de soustractions, de sélections, d'amplifications. L'œil du peintre, la métaphore du poète, la ligne mélodique du musicien se saisissent du récit et l'investissent de significations nouvelles, parfois en collant de près au texte, souvent en prenant une distance marquée par rapport à lui. Dans ce cadre, l'analyse narratologique, dont l'application aux textes bibliques est relativement récente, s'avère fort utile, puisqu'elle permet de fixer l'attention sur le texte lui-même et de secouer ainsi les représentations ou réécritures du texte portées par la mémoire collective, à travers les multiples reprises dont ce texte a été l'objet. Un autre avantage de l'analyse narratologique est qu'elle s'applique autant à un dispositif pictural qu'à un texte littéraire, permettant un travail de comparaison entre le dispositif narratif du texte biblique d'origine ${ }^{3}$ et le dispositif narratif de sa réécriture picturale ou littéraire. En révélant les mécanismes narratifs du texte, mais aussi ses ambiguïtés et son caractère le plus souvent plurivoque, une analyse narratologique aide à comprendre les points d'ancrage de la multiplicité des relectures que le récit a suscitées. Quels aspects narratifs du récit l'artiste a-t-il privilégiés ou, au contraire, occultés ? Quels silences a-t-il exploités? Quels ont été ses choix interprétatifs, ses partis pris, ses hypothèses qui, une fois injectées dans le texte, ont pu en remplir certains «trous»? Quel point de vue a-t-il adopté ?

Qu'une même méthode puisse s'appliquer au texte biblique et à ses reprises possède aussi un dernier avantage, celui de rendre possible et d'encourager un dialogue interdisciplinaire entre l'exégète biblique et, par exemple, l'historien de l'art. Spécifiquement, dans le cadre de ce numéro de Théologiques, je me propose ici de déployer certaines facettes narratives de Gn 4,1-16, pour éventuellement entrer en dialogue avec mon collègue Alain Laframboise, qui présentera ses analyses de quelques reprises picturales de l'histoire des deux frères, Caïn et Abel.

3. Si, dans la perspective adoptée ici, le texte biblique est à l'origine du mouvement de relectures, il n'est pas «originel». Tant le travail de comparatisme des grands mythes (dès le XIX ${ }^{e}$ siècle) que la sensibilité intertextuelle (fin $\mathrm{XX}^{\mathrm{e}}$ siècle), sans oublier la prise de conscience du lent processus de rédaction de la Bible, nous mettent en garde de faire du texte biblique un point de départ absolu. Le texte biblique est lui-même la réécriture de mythes empruntés à des civilisations avoisinantes ou la modification de traditions orales antécédentes. 


\subsection{Quelques lignes interprétatives de Gn 4}

De l'avis général, le récit de Gn 4 est lapidaire et elliptique, voire lacunaire. Plusieurs tournures surprenantes - qui seront relevées plus loin - attendent le lecteur attentif à ne pas aplanir les aspérités du texte. Particulièrement, on repère facilement des trous - des blancs, selon l'anglicisme narratologique - que les traductions, reprises ou commentaires du texte à travers l'histoire ont cherché à combler. À titre d'exemples, trois interrogations reviennent de manière récurrente: Pourquoi le Seigneur préfère-t-il l'offrande d'Abel ? (v. 4-5) Que dit Caïn à Abel avant de le tuer ? (v. 8) En quoi consiste la marque de Caïn? (v. 15)

Quelques lignes interprétatives sont repérables:

- Dès la LXX et Philon d'Alexandrie (Rouillard 1986), les targums (Chilton 1982; Bassler 1986), puis le Nouveau Testament (Mt 23,35; He 11,4; Jude 11; 1Jn 3,12-134), la lecture moralisante s'est imposée (Kugel 1990). Cette lecture a été reprise en littérature française par le poème La conscience de Victor Hugo ${ }^{5}$. Caïn y prend les traits d'un homme aux mauvaises intentions, mesquin et rongé par l'envie, à la

4. He 11,4: «Par la foi, Abel offrit à Dieu un sacrifice meilleur que celui de Caïn. Grâce à elle, il reçut le témoignage qu'il était juste et Dieu rendit témoignage à ses dons. Grâce à elle, bien que mort, il parle encore "; Jude 11: "Malheur à eux, parce qu'ils ont suivi le chemin de Caïn; pour un salaire ils se sont abandonnés aux égarements de Balaam et ils ont péri dans la révolte de Coré»; 1Jn 3,12-13 : "Non comme Caïn: étant du Mauvais, il égorgea son frère. Et pourquoi l'égorgea-t-il ? Ses œuvres étaient mauvaises, tandis que celles de son frère étaient justes».

5. Le poème de Hugo, intégré à La légende des siècles, est une réécriture de la suite du récit de Caïn et Abel, soit Gn 4,17-24. L'image de l'œil (la conscience) est un ajout flagrant de Hugo. Les champs sémantiques du remords (livide, ne dormant pas, muet, pâle, frémissant, tressaillit, frisson, trembler), de la hâte dans la fuite sous le poids de la culpabilité (échevelé, enfui, hors d'haleine, fuir, marcha, furtif, sans regarder derrière, sans trêve, sans repos, sans sommeil) et des ténèbres (soir, sombre, funèbres, ténèbres, ombre, sinistre, nuits, sommeil, noir, cachez-moi, ne voyez plus rien, crever les yeux, clore, murer, sépulcre, fosse, souterrain, tombe) imprègnent les 68 vers. Hugo a eu pour devanciers Agrippa d'Aubigné et Gérard de Nerval, comme le souligne Hedwige Rouillard (1986), qui explique aussi que la lecture en termes de "fuite» s’appuie sur les glissements sémantiques de la LXX par rapport au TM, puis de Philon par rapport à la LXX:

TM: «Tu me chasses aujourd'hui de sur la face de l'humus et je serai caché de devant ta face».

LXX: «Si tu me chasses aujourd'hui loin de la face de la terre, aussi je serai caché loin de ta face».

Philon: "Si tu me chasses aujourd'hui de la face de la terre, moi aussi, je me cacherai loin de ta face». 
limite injuste et impie, surtout en comparaison de son frère Abel le juste (Lewis 1994; Kim 2001). Encore aujourd'hui, un certain discours homilétique et du matériel catéchétique adoptent cette voie (ainsi Greidanus 2004; Krašovec 1994).

- L'exégèse historico-critique du $\mathrm{XX}^{\mathrm{e}}$ siècle a eu tendance à expliquer le texte par sa fonction étiologique (Speiser, von Rad): Caïn serait l'ancêtre éponyme des Qénites (Gn 15,19). Encore récemment, Paula McNutt (1999, 45), à la lumière des études sociologiques sur les artisans dans l'Antiquité et en comparaison de mythes d'autres sociétés traditionnelles, regarde le récit comme "the representation of Cain and his descendants as culture heroes and as the eponymous ancestors of smiths and artisans ".

- S'articulant parfois sur le filon étiologique précédent, le récit de Caïn et Abel est interprété à la lumière du thème classique de la rivalité des deux frères (Cassuto 1961; Quinones 2004). Ainsi, il est possible de comparer le récit biblique à la légende de Remus et Romulus, pour montrer que toute civilisation semble être fondée sur la violence. Toutefois, comme le spécifie René Girard (1978, 210), même fondateur de la culture (comme le montre Gn 4,17-24), le meurtre d'Abel, contrairement à celui de Remus, n'est pas pour autant acceptable moralement et ne saurait être légitimé - du point de vue du narrateur biblique.

- Une nouvelle veine fort intéressante, qui s'appuie sur des observations narratologiques, s'est développée, principalement en contexte francophone (Beirnaert 1987; Basset 1998; Wénin 1999 et 2007). On pourrait la qualifier de psychanalytique. Caïn, enfermé dans une relation fusionnelle avec sa mère (qui a fait de son fils l'objet de son désir, de la même manière qu'elle a vu son propre statut de sujet dénié par son mari) ne peut accepter son manque et encore moins le dire, d'où sa colère fratricide. «Ainsi, ces pages illustrent une loi de l'humain: là où les forces du désir ne sont pas contenues, là où elles ne peuvent venir à la parole, elles tournent en convoitise qui envahit le sujet au détriment de lui-même et de l'autre» (Wénin 1999, 15).

D'emblée, on constate donc 1) l'extrême richesse des interprétations - ce qui appuie le caractère dialogique du texte;2) la constante tentation de réduire le texte à une seule lecture (monologique), car souvent ces interprétations se présentent comme exclusives l'une à l'autre.

Par ailleurs, on constate aisément que le contexte littéraire qu'on assigne au texte détermine la lecture - et le lien entre cette contextualisa- 
tion spécifique et les lignes interprétatives esquissées ci-dessus est parfois limpide:

- Un contexte biblique global, susceptible de couvrir les deux testaments chrétiens, opposera les deux descendances d'Adam: les descendants de Caïn ${ }^{6}$, fils de l'injustice ou du serpent, seront vaincus par les descendants de Seth, fils de la justice qui reprennent la lignée avortée d'Abel, et dont Abraham et plus tard Jésus sont les descendants (Greidanus 2004). On reconnaît ici la trame moralisante.

- Un contexte biblique, qui met en exergue l'élection gratuite de Dieu (au double sens de généreuse et arbitraire) en faveur du plus jeune frère, rapprochera Gn 4 d'autres passages qui portent cette thématique (Kim 2001, 67): Isaac et Ismaël (Gn 16.21), Jacob et Ésaü (Gn 27-28), Joseph et ses frères (Gn 37), Abimelech et les 70 frères (Jg 9), Amnon et Absalom (2S 13-14).

- Le contexte de la Torah permet de voir en Gn 4 une anticipation des nombreuses prescriptions qui ne seront données qu'avec Moïse, dont l'interdit du meurtre et les directives concernant les sacrifices. Gn 4 devient alors l'illustration de plusieurs grands principes de la Loi hébraïque (Wenham 1987, 117).

- Le contexte des mythes fondateurs de différentes cultures forme un corpus comparatiste qui interroge le lien entre civilisation et violence.

- On peut souligner le lien étroit qui existe entre Gn 4 et Gn 2-3, tant au plan de l'intrigue, du lexique que de la thématique (Wénin 2007, 135; van Wolde 1991, 25-26; Wenham 1987, 99 ; Hauser 1980). La lecture psychanalytique s'appuie entre autres sur ce découpage.

Ces diverses lignes interprétatives et contextualisations font partie de l'imaginaire occidental concernant "Caïn et Abel». Elles peuvent servir de point de repère pour situer une reprise littéraire, picturale ou musicale du mythe ${ }^{7}$. Comme déjà mentionné, elles ont pu influencer bien plus directement ces productions créatrices que le texte massorétique lui-même. Mais c'est le parti pris du présent article de retourner au texte, de s'y cantonner autant que faire se peut et de l'analyser à l'aide d'outils narratologiques. Jusqu'ici, on a pu mettre en valeur la construction des trois personnages Dieu — Caïn - Abel (van Wolde 1991; Zwilling 2005), la narration qui

6. Techniquement, la descendance de Caïn s'éteint au déluge; mais une dichotomie analogue s'établit entre les fils de Noé, particulièrement Sem et Cham.

7. Ainsi Michel Tournier, revisitant sous le mode de l'inversion les mythes de Genèse, réduit le couple Caïn et Abel à l'opposition sédentaire-terrestre et nomade-céleste (Beaude 2003, 435-437). 
conduit le lecteur de surprise en surprise (Zwilling 2005; Swenson 2006) et le cheminement psychologique de Caïn (Wénin 1999; 2007). Étrangement, l'intrigue et les prises de paroles successives ont été quelque peu négligées.

\section{Les prises de parole des personnages au fil de l'intrigue}

Gn 4,1-16 situe son action dans un «no man's land ", entre le jardin d'où est chassé l'humain et le pays de Nod où s'installera Caïn. Le récit a pour entête un déclencheur narratif: "L'humain connut Ève sa femme; elle conçut et enfanta Caïn» $(4,1)$, alors que le texte suivant a une entête semblable, qui marque donc une nouvelle péripétie: "Caïn connut sa femme et elle conçut et enfanta Hénok» $(4,17)$. Deux nouveaux personnages sont les protagonistes de Gn 4,1-16: Caïn et Abel. L'intrigue qui s'y dessine en confirmera l'unité: une succession d'initiatives divines qui sous-tendent des attentes de Dieu auxquelles Caïn répond peu ou prou. C'est en ce sens que je parlerai d'une suite d'épreuves proposées par Dieu à Caïn, qui constituent des rendez-vous manqués jusqu'à l'acte irréversible du fratricide et l'ultime confrontation qui s'ensuit.

Le tableau ci-dessous compare le découpage adopté dans cet article à deux autres découpages possibles. Dans les trois cas, le meurtre du v. 8 constitue le pivot de l'action. Gordon Wenham met en valeur la correspondance thématique entre les scènes 1 et 5 (narratives) et 2 et 4 (discursives), avec le résultat quelque peu statique selon lequel la deuxième partie confirme et accentue les données de la première partie: Caïn, dans son humanité marquée par l'animalité, ne réussit pas à être exaucé par Dieu ${ }^{8}$ Avec André Wénin, j'insiste plutôt sur le renversement et la transformation qui s'opèrent entre l'avant et l'après. La proposition de Wénin, de l'ordre du chiasme thématique, fait bien ressortir la transformation du récit, mais fragmente artificiellement l'ensemble du dialogue C'-D' (v. 9-15): Caïn passe de l'état de cultivateur à celui d'errant (affirmation qui sera à nuancer); le sol d'abord fertile devient stérile; Dieu cherche d'abord à prévenir le meurtre puis à faire prendre conscience de la portée du meurtre.

8. Bien que globalement suggestive, l'analyse de Wenham comporte plusieurs inexactitudes de détail: a) elle ne tient pas compte des v. $1-2 \mathrm{a}$; b) la scène 2 est en fait un monologue; c) le dialogue de la scène 4 se poursuit en $15 \mathrm{a}$; d) surtout, la thématique de la scène 5 serait plutôt que Dieu répond par le «marquage » à la demande de Caïn du v. 14. 


\begin{tabular}{|c|c|c|c|}
\hline & Wenham 1987, 99 & Wénin 2007, 134 & Gignac \\
\hline v. 1 & & \multirow{3}{*}{$\begin{array}{l}\text { A Naissance de Caïn } \\
\text { - cultivateur }\end{array}$} & \multirow[t]{3}{*}{ 1. Situation initiale } \\
\hline v. $2 \mathrm{a}$ & & & \\
\hline v. $2 b$ & \multirow{5}{*}{$\begin{array}{l}\text { Scène 1: Récit } \\
\text { Caïn s'approche de } \\
\text { Dieu (sacrifice) mais } \\
\text { n'est pas exaucé }\end{array}$} & & \\
\hline v. 3 & & \multirow{3}{*}{\begin{tabular}{|l} 
B Le sol produit \\
du fruit \\
- sacrifice
\end{tabular}} & \multirow[t]{6}{*}{ 2. Nouement } \\
\hline v. 4 & & & \\
\hline v. $5 \mathrm{a}$ & & & \\
\hline v. $5 \mathrm{~b}$ & & \multirow{3}{*}{$\begin{array}{l}\text { C Adonaï parle } \\
\text { avec Caïn pour le } \\
\text { faire fléchir }\end{array}$} & \\
\hline v. 6 & \multirow{2}{*}{$\begin{array}{l}\text { Scène 2: Dialogue } \\
\text { (tronqué) - péché } \\
\text { comme une bête tapie }\end{array}$} & & \\
\hline v. 7 & & & \\
\hline v. 8 & $\begin{array}{l}\text { Scène 3: (Dialogue) } \\
\text { récit }\end{array}$ & $\begin{array}{l}\text { D Meurtre (sans } \\
\text { parole) }\end{array}$ & 3. Point tournant \\
\hline v. 9 & \multirow{7}{*}{$\begin{array}{l}\text { Scène } 4 \text { : Dialogue } \\
\text { - la terre ouvre la } \\
\text { gueule pour boire le } \\
\text { sang }\end{array}$} & \multirow{2}{*}{$\begin{array}{l}\text { C'Adonaï dialogue } \\
\text { avec Caïn à propos } \\
\text { du meurtre }\end{array}$} & \multirow[t]{8}{*}{ 4. Dénouement } \\
\hline v. 10 & & & \\
\hline v. 11 & & \multirow{2}{*}{$\begin{array}{l}\text { B' Le sol ne produit } \\
\text { plus de fruit }\end{array}$} & \\
\hline v. $12 \mathrm{a}$ & & & \\
\hline v. $12 \mathrm{~b}$ & & \multirow{6}{*}{$\begin{array}{l}\text { A' Caïn errant sans } \\
\text { terre } \\
\text { - sortie }\end{array}$} & \\
\hline v. 13 & & & \\
\hline v. 14 & & & \\
\hline v. $15 \mathrm{a}$ & \multirow{3}{*}{$\begin{array}{l}\text { Scène } 5 \text { : Récit } \\
\text { Caïn s'approche de } \\
\text { Dieu (prière) mais n'est } \\
\text { pas exaucé [sic] et } \\
\text { finalement s'éloigne }\end{array}$} & & \\
\hline v. $15 \mathrm{~b}$ & & & \multirow[t]{2}{*}{ 5. Situation finale } \\
\hline v. 16 & & & \\
\hline
\end{tabular}

\subsection{Situation initiale}

${ }^{1}$ L'Humain connut Ève sa femme. Elle conçut et enfanta Caïn et dit: "J'ai acquis un homme avec le SEIGNEUR. » ${ }^{2}$ Elle ajouta à l'enfantement [ou: elle continua à enfanter] son frère Abel. Abel devint berger de moutons, tandis que Caïn devint serviteur de l'humus (traduction TOB modifiée).

L'Humain, Ève, Caïn, le Seigneur, Abel. L'ouverture du récit met rapidement en scène cinq personnages, dont les deux premiers, l'Humain et sa femme Ève, sont des personnages ficelles qui permettent au lecteur de rattacher Gn 4 à ce qui précède. L'Humain (v. 1) désigne ici l'Adam, avec article, lié depuis Gn 2,5.7.15; 3,179 à l'humus (v. 2) — un jeu de mots qui rend le rapprochement entre les deux termes hébraïques 'ādām et ${ }^{\prime a} \underline{d} \bar{a} m a \hat{a}$. L'humus,

9. Ces trois mentions conjuguées de «humain - humus » ont une fonction proleptique par rapport à notre récit. Selon Gn 2,5.7.15, l'humain est tiré de l'humus pour le "servir» (le cultiver) — ce que confirme Gn 4,2-3; mais, selon Gn 3,17-19, le rapport entre l'humain et l'humus sera problématique, marqué par la malédiction - ce que réalisera voire accentuera Gn 4,12. 
d'abord un lieu qui caractérise Caïn, deviendra un enjeu et même un sixième personnage à part entière aux v. 11-14. Si l'humain-Adam disparaît de la scène aussitôt qu'apparu, il n'en va pas de même pour Ève. Celleci parle la première dans un texte qui n'est pas avare de paroles. En prenant la parole, Ève donne un sens surprenant à son enfantement: elle donne naissance à un homme (et non à un fils, ou à un enfant), s'accapare cet homme jusqu'à sous-entendre par un jeu de mots que son nom, Caïn, signifie "Acquisition ${ }^{10}$ ", et évacue son homme (Adam) en introduisant dans sa relation conjugale un tiers, le Seigneur. Littéralement, c'est elle qui fait disparaître Adam de l'histoire et qui y fait entrer le personnage «le Seigneur». C'est la mère, et non le narrateur, qui prononce ce qui s'avérera au fil de la lecture le double "programme ${ }^{11}$ " de Caïn: un personnage qui affrontera le problème de l'avoir et aura une relation particulière avec le Seigneur.

D'emblée, l'identité de Caïn est trouble... et troublante. Quel est son père? D'une part, le narrateur désigne l'Humain comme le géniteur de Caïn et spécifie que, tout comme ce géniteur, Caïn est lié à l'humus pour le «servir ». D'autre part, Ève présente un autre point de vue et assigne à Caïn un pro-créateur (ou co-créateur) divin.

Le narrateur acquiesce à la logique d'acquisition d'Ève, en disant que celle-ci ajoute à sa maternité un second fils. Au contraire de la première naissance, aucune parole pour dire le sens de l'arrivée d'Abel, sinon le nom très évocateur que le narrateur (et non la mère) lui attribue, puisque que ce nom vient d'une racine hébraïque qui signifie vapeur, fumée. Abel, d'entrée de jeu, manque de consistance. Si Caïn est caractérisé par son rapport au Seigneur, Abel est caractérisé par son rapport à Caïn. Outre son métier de berger et le fait que le Seigneur regarde son offrande, tout ce que l'on saura d'Abel est qu'il est le frère de Caïn (l'expression revient sept fois).

Bref, la première scène du récit campe succinctement et efficacement les protagonistes du drame à venir, que rien ne laisse présager.

\subsection{Nouement}

${ }^{3}$ À la fin des jours, Caïn apporta au SEIGNEUR une offrande de fruits de l'humus, ${ }^{4}$ tandis qu'Abel apporta lui aussi des prémices de ses bêtes et leur

10. L'assonance de la traduction française «Caïn / acquis " rend bien le rapprochement hébraïque: qayin et qānîtî.

11. Me situant en narratologie, je n'emploie pas le terme «programme» en son sens technique sémiotique (conjonction ou disjonction d'avec un objet-valeur), mais plutôt comme une parole qui donne une clé (pour l'instant cryptée) du destin du personnage Caïn. 
graisse. Le SEIGNEUR regarda vers Abel et son offrande, ${ }^{5}$ mais il ne regarda pas vers Caïn et son offrande. Caïn en fut très irrité et sa face tomba. ${ }^{6} \mathrm{Le}$ SEIGNEUR dit à Caïn :

— «Pourquoi t'irrites-tu ? Et pourquoi ta face est-elle tombée ? ${ }^{7} \mathrm{Si}$ tu agis bien, n'y aura-t-il pas élévation? Si tu n'agis pas bien, à ta porte Péchée est embusqué et vers toi sa convoitise: mais toi, ne la domineras-tu pas?»

Le texte introduit deux motifs qui prendront une grande importance. D'une part, il est question de visage et de regard ${ }^{12}$ : du fait de ne pas être regardé (v. 5), la face de Caïn s'affaisse (v. 5-6, voir van Wolde 1991, 32). Or, le mot face (pānîm, au pluriel en hébreu) reviendra dans la seconde partie du texte: la face de l'humus (v. 14), la face du Seigneur (v. 14.16). D'autre part, la spatialité haut/bas commence à structurer le texte: la face de Caïn tombe, mais il demeure une possibilité d'élévation; Péchée est embusqué ${ }^{13}$, comme tapi au sol, mais prêt à bondir, dirigeant son désir vers Caïn - c'est pourquoi Caïn doit la dominer. Plus loin, Caïn se dressera contre Abel et le sang de celui-ci criera depuis l'humus.

Le drame se noue autour d'une initiative de Caïn aux conséquences imprévues. Le cultivateur offre au Seigneur les fruits de son travail, imité en cela par Abel. Or, son offrande n'est pas regardée par le Seigneur, sans que le récit apporte une justification explicite ${ }^{14}$. À s'en tenir au texte, la raison du refus divin est dérobée au lecteur - qui pourrait même avoir tendance à percevoir la décision du Seigneur comme arbitraire. Le narrateur, quant à lui, ne se prononce pas.

Une chose est claire: Caïn réagit par la colère et sa contenance s'écroule. Le narrateur nous livre ainsi la première et seule focalisation interne du récit - l'émotion éprouvée par Caïn - que confirme la description physique de la face de Caïn. L'émotion est somatisée, et le corps peut ainsi la révéler. La question verbalisée par le Seigneur confirme — et dédouble ${ }^{15}$ — l'information fournie par le narrateur quant à l'irritation de Caïn, mais elle a

12. Voir les développements de Levinas sur le visage et l'analyse qu'en fait Thérèse Nadeau-Lacour (1995).

13. L'hébreu est notoirement difficile: le substantif est féminin (Péchée), mais le participe qui s'y rattache est masculin. Entre autres, voir les explications de Wenham (1987, 104-106).

14. À moins de considérer l'offrande des prémices et de la graisse comme qualitativement supérieure, ou encore de caractériser les deux personnalités comme étant justes et injustes, ou de prêter à Caïn une moins bonne intention qu'à Abel — alternatives empruntées amplement par l'interprétation moralisante.

15. Selon Alter (1999, 123-155), lorsque le texte biblique, si économe de ses moyens, se répète, cela doit allumer une lumière rouge chez le lecteur. 
tout pour surprendre à nouveau le narrataire: «Pourquoi t'irrites-tu ? Et pourquoi ta face est-elle tombée? » Le narrataire, lui, sait bien pourquoi Caïn est irrité et il reconnaît facilement que cette réaction est normale (Wénin 2007, 143-144 parle même d'une stratégie pour que le lecteur s'identifie à Caïn, ou du moins prenne son parti). Pourquoi le Seigneur ne trouve-t-il pas la réaction de Caïn normale (Swenson 2006, 379) ? Chose certaine, il semble que la réaction d'irritation de Caïn ne corresponde pas aux attentes divines. Comme si le Seigneur, par le refus de l'offrande (ou du moins, sa décision de ne pas la regarder), avait voulu tester de façon un peu provocatrice Caïn, et que celui-ci n'avait pas su réagir de manière appropriée...

La suite du discours divin accentue l'énigme plutôt que d'y répondre. D'autant plus que la structure syntaxique et grammaticale du monologue des v. 6a-7 est laborieuse, en hébreu (van Wolde 1991, 30-32). On peut toutefois y voir une suite de quatre questions (Wénin 1999, 4), qui n'appellent pas de réponse mais sont plutôt de l'ordre de l'exhortation. De toute façon, Caïn n'y réagit pas. Un nouveau personnage est mentionné par le Seigneur: Péchée ${ }^{16}$ y prend l'allure d'une bête prête à sauter sur Caïn. L'irritation de Caïn est-elle ici personnifiée ? L'émotion fait-elle de Caïn un animal tueur? C'est bien ce qui se manifestera lorsque Caïn se dressera contre son frère, au verset suivant. Le Seigneur invite Caïn à dominer son animal...

Risquons une hypothèse en quatre points, pour caractériser l'ensemble du nouement (v. 3-7) comme le «déraillage» du programme d'Ève pour son fils (énoncé au v. 1). Premièrement, la relation particulière avec le Seigneur semble être déniée par la non-acceptation de l'offrande. Deuxièmement, Caïn-Acquisition semblait voir son offrande comme un marchandage. La réaction du Seigneur le déstabilise, comme une invitation à changer de registre. Caïn perd ce qu'il donne sans rien acquérir en retour. Troisièmement, Dieu décrit la relation entre Caïn et Péchée dans les mêmes termes qu'il avait utilisés pour décrire la relation entre Adam et Ève en Gn 3:

\begin{tabular}{|l|l|l|l|l|}
\hline $\begin{array}{l}\text { Gn 3,16 (Dieu parle à } \\
\text { Ève d'Adam): }\end{array}$ & Vers ton homme & ta convoitise; & lui & te dominera \\
\hline $\begin{array}{l}\text { Gn 4,7 (Dieu parle à } \\
\text { Cain de Péchée): }\end{array}$ & Vers toi & sa convoitise; & toi & $\begin{array}{l}\text { ne la domineras-tu } \\
\text { pas? }\end{array}$ \\
\hline
\end{tabular}

16. Influencé par ma lecture de $\operatorname{Rm} 5-8$ où Paul personnifie Péché, j’ose ici faire de même (Gignac et Viard 2010). 
Le programme d'Ève quant à l'ascendance de Caïn se trouve contrecarré. Là où Ève cherchait à évacuer Adam et à donner à Caïn le Seigneur pour père, celui-ci rappelle implicitement à Caïn qu'il doit suivre les traces de son père Adam. Ou du moins, occuper une posture, une fonction de domination semblable à celle d'Adam. Le lecteur doit-il comprendre que Caïn doit rejeter le programme énoncé pour lui par sa mère? Inversement, dans ce parallélisme bizarre, une analogie est suggérée entre Péchée et Ève - le personnage divin est-il en train de dévaloriser la mère de Caïn? Quatrièmement, le Seigneur semble mettre Caïn à l'épreuve, par son refus inexpliqué et inexplicable de l'offrande, par son étonnement face à la réaction pourtant légitime de Caïn et par sa série d'exhortations. Paradoxalement, cette mise à l'épreuve pourrait annuler les trois premières observations et constituer un renforcement du lien privilégié entre Caïn et le Seigneur. À moins qu'il ne s'agisse d'un déplacement radical qui implique un nouveau lien, un lien autre, autrement constitué ?

\subsection{Point tournant}

${ }^{8}$ Caïn dit à Abel son frère: «». Lorsqu'ils furent dans le pâturage, Caïn se dressa contre Abel son frère et le tua.

Élément significatif: c'est dans la campagne sauvage, dans le domaine d'Abel, que le premier meurtre se produit. Avec justesse, van Wolde (1991, 35) affirme que ce verset est "the most elliptically described murder in literature » : court, sobre, sans description, il fait contraste avec le monologue qui précède et le dialogue très élaboré qui suit — comme si le meurtre était moins important que le dialogue entre Dieu et Caïn (van Wolde 1991, 36). Il ne s'agit pas de la pointe du récit ou de son sommet, mais simplement d'un pivot où l'histoire bascule et où Caïn est incapable de maîtriser son irritation. Caïn échoue une seconde fois à satisfaire l'attente de Dieu, telle qu'elle avait été proposée dans les exhortations précédentes.

Surtout, quelque chose se joue dans le non-dit ou le refus de la parole. Caïn n'a rien répondu à l'interpellation du Seigneur des v. 6-7. Mais aussitôt, il parle à son frère Abel. Or, si le narrateur est capable de rapporter les propos du Seigneur, il ne peut ou ne veut pas nous informer de la conversation entre les deux frères (une lacune, rappelons-le, que la Septante et surtout les Targums se sont fait un plaisir de combler ${ }^{17}$ ). Abel, lui, ne dit rien. Ce n'est

17. Pamela Reis (2002) suggère de traduire wayyō'mer par « récrimina contre ", une sorte de parole réflexive, tournée sur elle-même, où Caïn ressasse plus pour lui-même que pour son frère sa frustration. Or, cette suggestion est loin de faire l'unanimité. 
qu'après le meurtre que la parole sera libérée, pour ainsi dire (chez Caïn et, à titre posthume, chez Abel, par son sang) - mais ce sera alors une parole marquée par la malédiction et le désespoir.

\subsection{Dénouement}

${ }^{9}$ Le SEIGNEUR dit à Caïn: «Où est Abel ton frère?»

— Il dit: «Je ne sais. Suis-je le gardien de mon frère?»

— ${ }^{10}$ Il reprit: "Qu'as-tu fait? La voix du sang de ton frère crie vers moi depuis l'humus. ${ }^{11}$ En conséquence tu es maudit. Depuis l'humus qui a ouvert la bouche pour recueillir le sang de ton frère de ta main. ${ }^{12}$ Quand tu serviras l'humus, il n'ajoutera plus pour toi sa force. Tu seras errant et vagabond sur la terre.»

— ${ }^{13}$ Caïn dit au SEIGNEUR: «Ma faute est trop lourde à porter. ${ }^{14}$ Voici que tu me chasses aujourd'hui de sur la face de l'humus. Alors de ta face je serai caché et je deviendrai errant et vagabond sur la terre et quiconque me trouvera me tuera.»

— ${ }^{15 a}$ Le SEIGNEUR lui dit: «Eh bien! Si l'on tue Caïn, il sera vengé sept fois. »

Au v. 9, le dialogue ne tarde pas à s'instaurer, mais sous le mode du jeu du chat et de la souris. La question de Dieu est soit sournoise (s'il connaît déjà la réponse, tout comme le lecteur), soit mal informée (s'il fait sa prise de conscience seulement au v. 10). Quoi qu'il en soit, cette question agit comme un leurre. Si la réponse de Caïn n'est pas strictement un mensonge (malgré la lecture moralisante de Greidanus 2004, 393), puisqu'il ne sait pas où se trouve Abel une fois mort, elle relève de l'esquive et verse dans l'ironie: «suisje le gardien de mon berger de frère? » À la question de Dieu, Caïn répond par une question mais ne recevra pas de réponse - comme s'il devait luimême répondre à sa question (ou comme si le narrateur invitait le lecteur à y répondre, Swenson 2006, 374). Une troisième fois, le Seigneur intervient pour confronter Caïn à l'épreuve et une troisième fois, par son refus explicite d'être gardien de son frère, Caïn échoue au test.

La quatrième intervention sera la bonne. Au v. 10, le personnage Dieu fait prendre conscience à Caïn — et du même coup, au lecteur — que son frère crie à partir de l'humus, ou du moins la voix de son sang. C'est la première fois que Abel parle: «as a dead man Abel is more active and present than when alive» (van Wolde 1991, 37). L'image qui suit est saisissante: tel un animal, l'humus a ouvert la gueule pour se nourrir du sang d'Abel. Voix 
du sang et gueule qui aspire le sang: les deux images se télescopent pour donner le lieu à partir duquel est lancée la malédiction: «depuis l'humus» (répété deux fois, v. 10 et 11). Si on ponctue les v. 10-11 en quatre membres (qui respectent l'atnah massorétique), on s'aperçoit que la malédiction peut se rattacher tout autant à ce qui précède (la voix du sang d'Abel) qu'à ce qui suit (la bouche de l'humus qui avale le sang ${ }^{18}$ ). Malgré toute la tradition de lecture qui prête au Seigneur la malédiction, il semble donc que celle-ci relève plutôt d'Abel et/ou de l'humus: le personnage divin ne fait que la constater et en faire prendre conscience à Caïn. Étrangement, c'est le programme divin pour Adam et sa descendance (Gn 3,17), et le programme d'Ève pour Caïn (Gn 4,1) qui se trouvent d'un même coup définitivement bloqués: "Quand tu serviras l'humus, il n'ajoutera plus pour toi sa force». Caïn-Acquisition ne pourra plus acquérir, ajouter à ce qu'il possède.

Cependant, Caïn va ajouter à sa culpabilité. Soudainement, au v. 13, après l'assourdissant cri du sang d'Abel et de l'humus, Caïn répond enfin franchement et de manière authentique au Seigneur: " ${ }^{a} w \bar{o} n \hat{\imath}$, ma sentence — mais aussi ma faute et mon remords (van Wolde 1991, 36; Wénin 2007, 156) - est trop lourde à porter ". Manifestement, Caïn en rajoute et affirme que le Seigneur le chasse de sur la face de l'humus. Jamais le Seigneur ne lui a dit une telle chose, par ailleurs impossible à réaliser. Il s'agit d'une interprétation propre à Caïn, qui poursuit dans son exagération en disant: «je serai caché à ta face» - une remarque à mettre en rapport d'analepse avec l'épisode du v. 5, lorsque le Seigneur n'avait pas regardé l'offrande de Caïn. Celui-ci déplace donc le point de vue: que le Seigneur ne regarde pas l'offrande n'est pas la même chose que de ne pas regarder Caïn, voire de lui tourner le dos ou de l'exiler le plus loin possible de lui. Caïn ajoute encore à son fardeau: outre l'errance mentionnée par Dieu, il affirme que son statut de meurtrier apatride lui vaudra de devenir la victime désignée du premier venu. Le Seigneur semble reconnaître la justesse de

18. On observe d'ailleurs aux 10-11 un remarquable concentrisme, dont la pointe émergente est la malédiction:

A Qu'as-tu fait?

$B$ La voix du sang de ton frère

C crie vers moi

$\mathrm{D}$ depuis l'humus.

E En conséquence tu es maudit.

D' Depuis [ou: loin de] l'humus

C' qui a ouvert la bouche

B' pour recueillir le sang de ton frère

A' de ta main.

Notons qu'on traduit généralement le v. 11: «Tu es maudit loin de l'humus...». 
l'affirmation, puisqu'il lui promet qu'il sera vengé, si cela devait arriver. L'utilisation du passif «être vengé » ou «il sera fait vengeance » permet de ne pas mentionner qui sera le vengeur. Faut-il en faire un passif divin? En ce cas, le Seigneur se ferait implicitement solidaire de Caïn, à la manière dont un clan est solidaire de chacun de ses membres.

En somme, le dénouement est paradoxal. À nouveau, le Seigneur a mis Caïn à l'épreuve. D'abord en lui demandant de porter attention à son frère — responsabilité que Caïn rejette, échouant à nouveau au test. Puis, en le plaçant devant les conséquences effrayantes de son acte effroyable. Mais pour la première fois, Caïn réussit alors le test, par sa prise de conscience et l'aveu de sa détresse. Alors que Caïn constate que le programme de sa mère en ce qui concerne sa relation au Seigneur a échoué: "Je serai caché à ta face ", le Seigneur répond à la détresse de Caïn (sans que celui-ci le lui ait demandé explicitement) en se faisant solidaire du vagabond, comme quelqu'un qui se porte garant de la vie d'un parent de son clan. Au moment où l'effritement du lien amorcé par le rejet de l'offrande et cristallisé par le meurtre est consommé par la malédiction, voilà que le Seigneur semble proposer un autre lien - un lien symbolisé par ailleurs par l'ensemble du dialogue dont Dieu a l'initiative et auquel Caïn finit par consentir. Malgré son meurtre (ou à cause de lui), Caïn parle enfin au Seigneur. Celui que la mère a privé de son père pour ensuite ne plus se préoccuper de lui, celui dont le frère est disparu, celui qui par trois fois n'a pas répondu aux attentes du Seigneur, celui qui est seul face à la malédiction, conserve la possibilité de parler au Seigneur - c'est-à-dire d'être en réelle relation avec lui.

\subsection{Situation finale}

${ }^{15 b}$ Le SEIGNEUR mit un signe sur Caïn pour que personne en le rencontrant ne le frappe. ${ }^{16}$ Caïn s'éloigna de la face du SEIGNEUR. Il habita dans le pays d'Errance (Nod) à l'orient d'Éden.

La chute du récit est sobre. Quelle que soit la nature du signe mis sur Caïn par le Seigneur ${ }^{19}$, sa signification est claire: il concrétise la parole de ven-

19. Relevons l'hypothèse récente de Moberly $(2007,15)$ : «When one takes these two factors together - that the text implies a non-corporeal mark to protect Cain from being killed, and that YHWH is introducing a general saying about the perilous outcome of killing Cain - then my thesis about the nature of the mark of Cain should, I hope, become apparent. It is the saying in the text, "Whoever kills Cain will suffer sevenfold vengeance," that is itself the non-corporeal sign, the warning, which serves to prevent Cain from being killed. YHWH's words to Cain and the sign are not two different things $[\ldots] »$. 
geance prononcée par le Seigneur et «marque» la nouvelle relation qui s'établit entre le Seigneur et Caïn, le second se trouvant en quelque sorte sous la protection du premier.

Le récit se termine par deux dernières surprises. D'une part, en contradiction à la remarque de Caïn selon laquelle il serait caché à la face du Seigneur - elle-même un ajout par rapport aux constats divins de la malédiction du sol et de l'errance du meurtrier —, le narrateur apporte une nuance importante: Caïn ne fait que s'éloigner de la face du Seigneur. D'autre part, le narrateur atténue voire annule la sentence d'errance sur laquelle Dieu et Caïn s'entendaient, en affirmant paradoxalement que Caïn «habita dans le pays d'Errance».

La chute du récit laisse donc le lecteur dans l'incertitude. Surtout, comme le souligne Alter $(1999,108)$ à propos du fonctionnement extrêmement économe des dialogues bibliques, les reprises des affirmations de Dieu par Caïn, puis des affirmations de Dieu et Caïn par le narrateur, dans leurs subtiles différences, s'avèrent significatives. Non seulement elles déstabilisent le lecteur, mais elles l'empêchent de clore le récit de façon monologique. On note ici trois voix différentes qui proposent autant de conclusions différentes au même récit:

\begin{tabular}{|c|c|c|c|c|}
\hline $\begin{array}{l}\text { Dieu } \\
\text { (v. 12) }\end{array}$ & $\begin{array}{l}\text { Quand tu serviras l'hu- } \\
\text { mus, il n'ajoutera plus } \\
\text { pour toi sa force. }\end{array}$ & - & \begin{tabular}{|l} 
Tu seras \\
errant et \\
vagabond \\
sur la terre.
\end{tabular} & $\longrightarrow$ \\
\hline $\begin{array}{l}\text { Caïn } \\
\text { (v. 13) }\end{array}$ & $\begin{array}{l}\text { Si tu me chasses } \\
\text { aujourd'hui de sur } \\
\text { la face de l'humus, }\end{array}$ & $\begin{array}{l}\text { je serai caché } \\
\text { à ta face. }\end{array}$ & $\begin{array}{l}\text { Je serai errant } \\
\text { et vagabond } \\
\text { sur la terre }\end{array}$ & $\begin{array}{l}\text { et quiconque me } \\
\text { trouvera me tuera. }\end{array}$ \\
\hline $\begin{array}{l}\text { Dieu } \\
\text { (v. 15a) }\end{array}$ & & & & $\begin{array}{l}\text { Si l'on tue Caïn, } \\
\text { il sera vengé sept } \\
\text { fois }\end{array}$ \\
\hline $\begin{array}{l}\text { Narrateur } \\
\text { (v. } 15 \mathrm{~b}- \\
16 \text { ) }\end{array}$ & $\longrightarrow$ & $\begin{array}{l}\text { Caïn s'éloigna } \\
\text { de la face du } \\
\text { SEIGNEUR }\end{array}$ & $\begin{array}{l}\text { Il habita } \\
\text { dans le pays } \\
\text { d'Errance } \\
\text { (Nod) }\end{array}$ & $\begin{array}{l}\text { Le SEIGNEUR } \\
\text { mit un signe sur } \\
\text { Caïn pour que } \\
\text { personne en le } \\
\text { rencontrant ne } \\
\text { le frappe }\end{array}$ \\
\hline
\end{tabular}

Autrement dit, le texte nous offre trois points de vue sur le sort de Caïn. Selon le point de vue de Dieu - réaliste et constatif ? - Caïn ne peut plus servir l'humus avec profit et deviendra vagabond, risquant de devenir victime à son tour (une éventualité soulevée en premier lieu par Caïn). Selon le point de vue de Caïn - pessimiste et exagéré ? -, c'est Dieu qui le coupe de l'humus et lui voile sa face, avec pour conséquence 
l'errance et le danger d'être tué. Selon le point de vue du narrateur - descriptif et ironique? - Caïn s'éloigne du Seigneur sans se cacher de lui, il habite un nouveau pays et court le risque d'être... frappé. Si, malgré des nuances, les points de vue de Caïn et Dieu correspondent (grosso modo), le point de vue du narrateur se distancie de celui des deux véritables protagonistes du récit. Au lecteur de trancher, ou de s'interroger ${ }^{20}$.

La situation finale prend donc le contrepoint de la situation initiale, en ce que Caïn est seul: l'Adam, Ève sa mère, Abel, l'humus, le Seigneur ont disparu de son écran radar. Plutôt que d'être cultivateur, le prochain récit nous apprendra qu'il est le fondateur de la première ville.

\section{Résultats}

Récit surprenant d'un meurtrier protégé par Dieu, d'un agriculteur dont l'offrande est refusée et dont la terre ne porte plus de fruit, et d'un errant qui habite au pays de l'errance (McNutt 1999, 45). À partir d'une approche narrative, j'ai pris le contre-pied de l'interprétation moralisante, de manière à retrouver un espace de liberté herméneutique et à démontrer que le texte biblique déstabilise nos idées préconçues sur Dieu et ouvre des chemins inusités. Dieu n'est pas tant un justicier ou le prête-nom d'une éthique moralisatrice, que celui qui veut entrer en dialogue avec l'humain et le faire accéder à la conscience morale. Or, ce Dieu est inventif dans sa manière d'agir sur Caïn, et tenace dans ses interventions plusieurs fois renouvelées pour faire «bouger» ou évoluer son interlocuteur — qui refuse par ailleurs longtemps de s'engager dans un réel «face à face ", c'est-à-dire dans un dialogue confrontant.

20. Si le lecteur endosse comme fiable le point de vue final du narrateur (objectivité), il pourra s'interroger sur la manière dont les deux personnages ont anticipé la fin de l'histoire (subjectivité des personnages) — incidemment, le «Dieu » mis en scène, s'il ne se trompe pas carrément sur la suite des choses (puisque Caïn, devenant citadin, cessera d'être agriculteur et s'implantera en pays d'Errance), n'est peut-être pas parfaitement omniscient. Si le lecteur choisit de ne pas faire aveuglément confiance à la version du narrateur — par exemple, dans une perspective (post)moderne —, il devra gérer la présence de trois versions d'un même récit, versions non nécessairement contradictoires mais éventuellement complémentaires. En définitive - mais ici on dépasse la frontière de la narratologie pour se situer «à l'heure du lecteur " (reader's response criticism) - , le lecteur devra prendre conscience de la version qu'il fait sienne et surtout des raisons pour lesquelles il le fait. 
Plusieurs voix prennent la parole, et elles expriment des programmes différents pour Caïn ou des évaluations discordantes de sa situation. "J'ai acquis un homme, avec le SEIGNEUR.» (v. 1) En ouverture du récit, Ève énonce un programme pour son fils Caïn que la suite va invalider. D'une part, Caïn-Acquisition perd son offrande, puis sa contenance (sa face tombe), puis son frère et la fécondité de l'humus. Négativement, il ne gagne ou n'accumule que des malédictions: errance, vagabondage, proscription, menace de mort. D'autre part, sa relation au Seigneur se détériore peu à peu, au point où il affirme: «je serai caché à ta face» (v. 14) et qu'il ne sera plus près du Seigneur. La face de l'humus et la voix du sang de son frère lui renvoient par ailleurs un cri de malédiction.

Pourtant, le fil conducteur de l'intrigue et la conclusion du récit offert par le narrateur contredisent cette conclusion apparente (qui semble assumée par Caïn). Tout au long du récit, le Seigneur porte attention à Caïn y compris par son choix initial et déclencheur d'ignorer l'offrande de Caïn! Le Seigneur lui parle, l'exhorte, l'interroge, le confronte et finalement le réconforte et le protège, en dépit de son meurtre. "In 4.15, YHWH gives Cain a mark of protection, and thus the very last action of YHWH shows that the link between Cain and YHWH is not totally broken off, but reduced to a minimum» (van Wolde 1991, 28). Une série d'initiatives montrent bien que Dieu talonne Caïn pour le forcer à réagir et à parler - à entrer en relation:

\begin{tabular}{|l|l|}
\hline \multicolumn{1}{|c|}{ Initiative divine } & \multicolumn{1}{c|}{ Réaction de Caïn } \\
\hline 1) Refus de l'offrande & Irritation (échec implicite) \\
\hline 2) «Domine Péchée» & Meurtre (échec explicite) \\
\hline 3) « Où est ton frère? » & «Suis-je le gardien de mon frère (échec) \\
\hline 4$)$ «Entends la malédiction» & «Ma sentence / faute est trop lourde» \\
\hline
\end{tabular}

Il est remarquable que la répétition des occasions manquées de correspondre aux attentes du Seigneur n'empêche pas que de nouvelles possibilités soient constamment offertes. Malgré la succession d'impairs, l'avenir n'est jamais fermé. Avant le fratricide, Caïn ne parle pas. C'est seulement après le meurtre qu'il accède au statut plénier de «personnage moral », qu'il prend la parole et qu'il entre en relation avec Dieu, capable de verbaliser sa faute et sa détresse. La relative neutralité du narrateur confirme cette lecture surprenante: "Caïn s'éloigna de la face du SEIGNEUR. Il habita dans le pays d'Errance (Nod) à l'orient d'Éden» (v. 16). Si, du point de vue de Caïn (et de sa mère), le projet généalogique d'engendrement n'existe plus, 
du point de vue du Seigneur et du narrateur, la possibilité d'une autre (et véritable) filiation se profile ${ }^{21}$.

On est loin ici de la lecture moralisante, tellement plus facile, qui conforte notre image du meurtrier méchant qui n'a que ce qu'il mérite avec l'image de Dieu correspondante. Les personnages de Caïn et du Seigneur sont déroutants, imprévisibles, en cheminement et... terriblement humains! Le meurtre - bien que terrible en soi — n'est pas la scène centrale du récit. C'est plutôt la relation qui s'établit entre Caïn et Dieu qui est le moteur de l'intrigue.

Pour reprendre le distinguo classique de la narratologie, en contrepoint d'une intrigue de résolution qui met en scène une rivalité fraternelle liquidée par un meurtre, on a pu mettre en relief une intrigue de révélation où le personnage Caïn accède pleinement à son identité morale, au fil de plusieurs tests qui lui permettent de déconstruire puis de construire sa relation à Dieu. Dans le premier cas, on a parlé traditionnellement du récit de Caïn et Abel, mais on devrait peut-être proposer un nouveau titre: Caïn et le Seigneur.

En terminant, pour revenir au dialogue interdisciplinaire et à la Wirkungsgeschichte, on pourra encore poser une question: l'histoire des représentations picturales de l'épisode de Caïn et Abel reflète-t-elle une lecture monologique du texte, ou permet-elle aussi de risquer une diversité d'interprétations? Quelles voix ces représentations laissent-elles entendre? Quel point de vue empruntent-elles? Focalisent-elles sur le meurtre, sur la culpabilité de Caïn ou sur le long cheminement d'un personnage qui accède en sa totalité à la responsabilité morale ${ }^{22}$ ?

\section{Références}

ALTER, R. (1999) [anglais 1981], L'art du récit biblique, Bruxelles, Éditions Lessius (Le Livre et le rouleau; 4).

BAKHTine, M. (1978), Esthétique et théorie du roman, Paris, Gallimard.

BASSET, L. (1998), "Où est ton frère "souffle” ", Études théologiques et religieuses, 73, p. 321-331.

21. On retrouve ce même déplacement dans la trajectoire de Paul de Tarse racontée en Galates 1-2, qui coupe avec sa généalogie paternelle et maternelle pour s'ouvrir à une filiation semblable à celle du Fils (Gignac 2008).

22. Je remercie les professeurs Robert David et André Wénin pour leur lecture attentive et leurs remarques suggestives. Le résultat final n'engage toutefois que l'auteur. 
Bassler, J. M. (1986), "Cain and Abel in the Palestinian Targums: A Brief Note on an Old Controversy ", Journal for the Study of Judaism, 17, p. 56-64.

BEaude, P.-M. (2003), «Tournier et le détournement du mythe biblique », Laval théologique et philosophique, 59, p. 421-439.

BEIRNAERT, L. (1987), «La violence homicide. L'histoire de Caïn et Abel (Genèse 4,1-25) ", dans Aux frontières de l'acte analytique. La Bible, saint Ignace, Freud et Lacan, Paris, Seuil, p. 186-194.

Brodie, T. L. (2001), Genesis as Dialogue: A Literary, Historical, and Theological Commentary, Oxford, Oxford University Press.

Cassuto, U. (1961), A Commentary on the Book of Genesis, Jérusalem, Magnes Press.

Chilton, B. (1982), «A Comparative Study of Synoptic Development: The Dispute between Cain and Abel in the Palestinian Targums and the Beelzebul Controversy in the Gospels ", Journal of Biblical Literature, 101, p. $553-562$.

ÉCole biblique de Jérusalem (19984), La Bible de Jérusalem, Paris, Cerf.

Hauser, A. J. (1980), «Linguistic and Thematic Links between Genesis 4:1-16 and Genesis 2-3", Journal of Evangelical Theological Studies, 23, p. 297305.

Genette, G. (1982), Palimpsestes. La littérature au second degré, Paris, Seuil (Points Essais; 257).

GignaC, A. (2008), «Une filiation sans engendrement? Ou le corps marqué de la lettre aux Galates ", dans M. Allard, D. Couture et J.-G. NADEAU, dir., Pratiques et constructions du corps en christianisme, Montréal, Fides (Héritage et projet; 75), p. 107-130.

Gignac, A. ET J.-S. Viard (2010), «Péché, Mort et Loi en Rm 5-8. Mises en intrigue et caractérisation ", dans A. PASQUIER, D. MARGUERAT et A. WÉNIN, dir., L'intrigue dans le récit biblique. Quatrième colloque international du RRENAB, Université Laval, Québec, 29 mai-1 ${ }^{e r}$ juin 2008, Leuven, Peeters (Bibliotheca Ephemeridum Theologicarum Lovaniensium; 237), p. 323-340.

Girard, R., J.-M. Oughourlian et G. Lefort (1978), Des choses cachées depuis la fondation du monde, Paris, Grasset.

GReIDANUS, S. (2004), «Preaching Christ from the Cain and Abel Narrative», Bibliotheca Sacra, 161, p. 387-397. 
Jenny, L. (2003), "Méthodes et problèmes. Dialogisme et polyphonie », $<$ http://www.unige.ch/lettres/framo/enseignements/methodes/dialogisme/ dpintegr.html> (consulté le 25 janvier 2009).

KIM, A. Y. (2001), "Cain and Abel in the Light of Envy: A Study in the History of the Interpretation of Envy in Genesis 4.1-16 ", Journal for the Study of the Pseudepigrapha, 12, p. 65-84.

Krašovec, J. (1994), "Punishment and Mercy in the Primeval History (Gen 1-11) ", Ephemerides theologicae lovanienses, 70, p. 5-33.

KugeL, J. L. (1990), "Cain and Abel in Fact and Fable: Genesis 4:1-16», dans R. Brooks, dir., Hebrew Bible or Old Testament? Studying the Bible in Judaism and Christianity, Notre Dame, Univ. of Notre Dame Press (Christianity and Judaism in Antiquity; 5), p. 167-190.

LEWIS, J. P. (1994), "The Offering of Abel (Gen 4:4): A History of Interpretation ", Journal of Evangelical Theological Studies, 37, p. 481-496.

McNutT, P. M. (1999), "The Land of Nod: Cain and Marginality ", Proceedings - Eastern Great Lakes and Midwest Biblical Societies, p. 1-25.

Moberly, R. W. L. (2007), "The Mark of Cain - Revealed at Last?", Harvard Theological Review, 100, p. 11-28.

Nadeau-Lacour, T. (1995), "L'impossible dérobade. Les voies de l'éthique selon E. Levinas ", dans C. MÉnARD et F. Villeneuve, dir., Drames humains et foi chrétienne. Approches éthiques et théologiques, Montréal, Fides (Héritage et projet; 55), p. 277-293.

Quinones, R. J. (2004), "The Cain-Abel Syndrome: in Theory and in History ", dans J.H. ElLENS, dir., Destructive Power of Religion Volume 3, Models and Cases of Violence in Religion, Westport/Londres, Praeger, p. 81-125.

ReIs, P. (2002), Reading the Lines: A Fresh Look at the Hebrew Bible, Peabody, Hendrickson.

Rouillard, H. (1986), «Et si Caïn voulait que l'œil le regardât: Étude des transformations de Gen 4, 14 à travers la LXX et Philon d'Alexandrie », dans Hellenica et Judaica, Leuven, Peeters, p. 79-83.

SARrazin, B. (1993), La Bible parodiée, Paris, Cerf.

SOCIÉTÉ BIBLIQUE FRANÇAISE (2004), La Bible: traduction cecuménique: édition intégrale, comprenant introductions générales et Pentateuque révisés, Paris/Villiers-le-Bel, Cerf/Société biblique française.

Swenson, K. M. (2006), "Care and Keeping East of Eden: Gen 4:1-16 in light of Gen 2-3», Interpretation, 60, p. 373-384. 
Wenham, G. J. (1987), Genesis 1-15, Waco, Word Books (Word Biblical Commentary).

WénIN, A. (1999), «Adam et Ève: la jalousie de Caïn, "semence” du serpent: un aspect du récit mythique de Genèse 1-4", Revue des sciences religieuses, 73, p. 3-16.

(2007), "Caïn et sa descendance Genèse 4 », D’Adam à Abraham, ou, Les errances de l'humain: lecture de Genèse 1,1-12,4, Paris, Cerf (Lire la Bible; 148), p. 131-165.

Wolde, E. J. van (1991), «The Story of Cain and Abel: A Narrative Study", Journal for the Study of the Old Testament, p. 25-41.

Zwilling, A.-L. (2005), "Caïn versus Abel (Gn 4,1-16) », dans C. FolCANT et A. WÉNIN, dir., Analyse narrative et Bible, Leuven, Leuven University Press / Peeters (Bibliotheca Ephemeridum Theologicarum Lovaniensium; 191), p. 507-516.

\section{Résumé}

La Bible interdit de représenter Dieu «en image» mais ne se prive pas de le mettre en scène dans des récits. Comment alors éviter que le lecteur ne se fabrique une «image de Dieu narrative » idolâtre? À partir du récit de Caïn et Abel (Gn 4), analysé sous l'angle des dialogues et des points de vue différenciés des personnages, l'article démontre que la narration biblique, par la manière dont elle raconte, brouille la représentation de Dieu et invite à une lecture dialogique. Loin de la lecture moralisante et culpabilisante traditionnelle (qui reprend uniquement le point de vue de Caïn), on se rend compte que le récit insiste sur les multiples initiatives divines pour entrer en discussion avec Caïn afin de le faire advenir réellement à lui-même, comme individu moralement responsable. En introduction, une réflexion est proposée quant à l'intérêt de la narratologie pour évaluer le jeu des multiples relectures artistiques d'un récit biblique et pour établir un dialogue interdisciplinaire entre l'exégèse et l'histoire de l'art.

\section{Abstract}

The Bible forbids any human made representation of God in the form of an image but it does present him as an actor in the narratives. How then can we avoid the fact that the reader can make for him/herself an idolatrous image of God? This article analyses the story of Cain and Abel (Gen 4) 
from the point of view of the dialogues and of the different viewpoints of the actors. It shows that be biblical narrative muddles up the representation of God and thus paves the way for a dialogical reading. Far from the traditional reading which interprets the text in a moral way while inducing a guilt feeling (which only takes into account Cain's point of view), it can be seen that the narrative insists on the multiple initiatives of God in order to create a dialogue with Cain so that he may become really himself, as an morally responsible individual. The introduction proposes a reflection on the interest of narratology to evaluate the reading of the multiple artistic interpretations of a biblical narrative and to establish an interdisciplinary dialogue between exegesis and the history of art. 